\title{
TANGENT MODELS AS A MATHEMATICAL FRAMEWORK FOR DYNAMIC CALIBRATION
}

\author{
RENÉ CARMONA AND SERGEY NADTOCHIY \\ BENDHEIM CENTER FOR FINANCE, ORFE \\ PRINCETON UNIVERSITY \\ PRINCETON, NJ 08544 \\ RCARMONA@PRINCETON.EDU \& SNADTOCH@PRINCETON.EDU
}

\begin{abstract}
Motivated by the desire to integrate repeated calibration procedures into a single dynamic market model, we introduce the notion of tangent market model in an abstract set up, and we show that this new mathematical paradigm accommodates all the recent attempts to study consistency and absence of arbitrage in market models. For the sake of illustration, we concentrate on equity models and we assume that market quotes provide the prices of European call options for a specific set of strikes and maturities. While reviewing our recent results on dynamic local volatility and tangent L'evy models, we provide new results on the short time-to-maturity asymptotics which shed new light on the dichotomy between these two disjoint classes of models, with and without jumps, helping choose in practice, which class of models is most appropriate to the market characteristics at hand.
\end{abstract}

\section{INTRODUCTION}

As a matter of motivation, we first start with a short discussion of the standard example of short interest rate used routinely as a justification for the introduction of the HJM approach to fixed income models. If we consider Vasicek's model for example

$$
d r_{t}=\kappa\left(\bar{r}-r_{t}\right) d t+\sigma d W_{t},
$$

because of the linear and Gaussian nature of the process, it is possible to derive explicit formulas for many derivatives and in particular for the forward and yield curves. However, the term structure given by these formulas is too rigid, and on most days, one cannot find reasonable values of the 3 parameters $\kappa, \bar{r}$ and $\sigma$ giving a theoretical forward curve matching, in a satisfactory manner, the observed forward curve $\tau \hookrightarrow f(\tau)$. This is a serious shortcoming as, whether it is for hedging and risk management purposes, or for valuing non-vanilla instruments, using a model consistent with the market quotes is imperative. Clever people found a fix to this hindrance: replace the constant parameter $\bar{r}$ by by a deterministic function of time $t \hookrightarrow \bar{r}(t)$. Indeed, this function being deterministic, the interest rate process remains a Gaussian process (at least as long as we do not change the initial condition) and we can still obtain explicit formulas for the forward curves given by the model. Moreover, it turns out that if we choose the time dependent parameter to be given by

$$
\left.\bar{r}(\tau)=f^{\prime}(\tau)+\kappa f(\tau)-\frac{\sigma^{2}}{2 \kappa}\left(1-e^{-\kappa \tau}\right)\left(3 e^{-\kappa \tau}-1\right)\right)
$$

Date: April 15, 2010. 
then the model provides a perfect match to the curve observed on the market! match, Our contention is that even though it provides a stochastic differential equation (SDE for short)

$$
d r_{t}=\kappa\left(\bar{r}(t)-r_{t}\right) d t+\sigma d W_{t},
$$

this procedure can mislead the casual newcomer who may be fooled into believing that this SDE actually relates to the dynamics of the short interest rate. Indeed, this is not a model in the sense that when the next day comes along, one has to restart the whole calibration procedure from scratch. Formula (1) does not provides a dynamic model, it is a mere artifact designed to capture the prices observed on the market.

One of the nagging challenges of quant groups supporting equity trading is to be able to generate Monte Carlo scenarios of implied volatility surfaces which are consistent with historical observations while being arbitrage free at the same time. Section ?? presents a solution based on a set of simple examples introduced in [2].

In a recent technical report [?], Kallsen and Krühner study a form of Heath-Jarrow-Morton approach to dynamic stock option price modeling. While similar in spirit to the tangent Lévy model approach of [?], it does not seem to lead to constructive models like the one proposed in Section ??.

\section{Tangent Models and Calibration}

Market Models for Equity Derivatives: problem formulation. We now describe the framework of the paper more precisely. First of all, as it is done in a typical set-up for a mathematical model, we assume that we are given a stochastic basis $\left(\Omega, \mathcal{F},\left(\mathcal{F}_{t}\right)_{t \geq 0}, \mathbb{Q}\right)$ and that pricing is linear in the sense that the time $t$ prices of all contingent claims are given as (conditional) expectations of discounted payoffs under the pricing measure $\mathbb{Q}$, with respect to the market filtration $\mathcal{F}_{t}$. We assume, for simplicity, that the discounting rate is one, and unless otherwise specified, all stochastic processes are defined on the above stochastic basis and $\mathbb{E} \equiv \mathbb{E}^{\mathbb{Q}}$. We denote by $\left(S_{t}\right)_{t \geq 0}$ the true risk-neutral (stochastic) dynamics of the price of the index or security underlying the derivatives in a specified set of liquidly traded instruments: in the present case, European call options for all strikes $K$ and maturities $T$. So at each time $t \geq 0$, we denote by

$$
\mathcal{D}_{t}=\left\{C_{t}(T, K)\right\}_{T>t, K>0}
$$

the set of all these prices. Our goal is to describe explicitly a large class of time-consistent market models, i.e. stochastic models (say, SDE's) giving the joint arbitrage-free time evolution of $S$ and $\mathcal{D}$. In other words, one would like to be able to start the model from "almost" any initial condition, typically the set of currently observed market prices, and prescribe "almost" any dynamics for the model provided it doesn't contradict the no-arbitrage property. Of course, the above formulation of the problem is rather idealistic. This explains our use of the word "almost" whose specific meaning is different for each classes of the market models we describe here.

The need for financial models consistent with the observed option prices comes mostly from the fact that call options have become liquid and provide reliable price signals to market participants. Stochastic volatility models (e.g. Hull-White, Heston, etc.) are very popular tools to capture this signal, but unfortunately, they do not reproduce market prices for all the strikes and maturities: their fit of the implied volatility surface is not always satisfactory. 
The preferred solution for over 15 years has been based on the so-called local volatility models introduced by Bruno Dupire in 1994. It essentially says that, assuming for simplicity zero interest rates, if the true model for the risk neutral dynamics of the underlying is given by an equation of the form $d S_{t}=\sigma_{t} d W_{t}$. then the stochastic process $\tilde{S}$ solving the equation

$$
\tilde{S}_{t}=S_{0}+\int_{0}^{t} \tilde{S}_{u} \tilde{a}\left(u, \tilde{S}_{u}\right) d W_{u}
$$

with

$$
\tilde{a}^{2}(T, K):=\frac{2 \frac{\partial}{\partial T} C(T, K)}{K^{2} \frac{\partial^{2}}{\partial K^{2}} C(T, K)},
$$

produces at time $t=0$, the same exact call prices $C(T, K)$ ! In other words, for all $T>0$ and $K>0$, we have:

$$
\mathbb{E}\left(\tilde{S}_{T}-K\right)^{+}=C(T, K)
$$

Notice that, here and in the following, we assume that the interest rate is 0 . This hypothesis plays little or no role in our considerations, and it simplifies the formulas and the exposition.

In the terminology which we develop below, the artificial financial model given by the process $\left(\tilde{S}_{t}\right)_{t>0}$, introduced for the sole purpose of reproducing the prices of options at time zero (in other words, the result of calibration at time zero), is said to be tangent to the true model $\left(S_{t}\right)_{t \geq 0}$ at $t=0$.

Tangent Models. One of the major problems with the calibration approach presented above is the frequency of the recalibration. For example, stochastic volatility models have different "optimal" parameters most every day, and the local volatility surface calibrated on a daily basis changes as well. So we focus on the temporary processes used to capture the price signals given by the market through the quotes of the liquidly traded vanilla options.

In what follows, we use the generic notation $\mathcal{D}_{t}$ for the chosen set of liquidly traded securities / derivatives written on the underlying $S$, and we add the subscript $t$ to emphasize the time when these derivatives are quoted (the set may change as, for example, some options expire). We use the notation $h$ for a typical payoff function of a derivative in $\mathcal{D}_{t}$ ( $h$ may depend upon the whole path of $S$ ) and $P_{t}(h)$ for the price at time $t \geq 0$ of the derivative with payoff $h(S)$ :

$$
P_{t}(h):=\mathbb{E}\left(h(S) \mid \mathcal{F}_{t}\right)
$$

We restate the result of the static exact calibration demonstrated above in the following way: a stochastic model given by $\left(\tilde{S}_{u}\right)_{u \geq 0}$ defined on a stochastic basis $(\tilde{\Omega}, \tilde{\mathcal{F}}, \tilde{\mathbb{P}})$ is $\mathcal{D}_{t}$-tangent to the true model (or just tangent when no ambiguity is possible) at time $t$ if, almost surely, we have

$$
\forall h \in \mathcal{D}_{t} \quad P_{t}(h)=\mathbb{E}^{\tilde{\mathbb{P}}}\left(h\left(\bar{S}^{t}\right)\right), \quad \text { where } \quad \bar{S}_{u}^{t}=\mathbf{1}_{u<t} S_{u}+\mathbf{1}_{u \geq t} \tilde{S}_{u} .
$$

Notice that $\bar{S}^{t}$ is introduced only because we allow payoff $h$ to depend upon the entire path of the underlying, however, in all particular constructions we will deal with the payoffs that only depend on $S_{T}$, for some maturity $T$, and in that case $\bar{S}^{t}$ can be replaced by $\tilde{S}_{T}$ in the above definition.

We want to think of the above notion of tangent model as an analog of the notion of tangent vector in classical differential geometry: the two models are tangent in the sense that, locally, at a fixed moment of time, they produce the same prices of derivatives in a chosen family. Recall that tangent vectors in differential geometry are often used as a more convenient way of describing the 
time dynamics. In the same way, one may hope that tangent models introduced above can help in solving the problem of market models formulated in the previous subsection.

Assume that we can find and parameterize explicitly a class of "simple" martingale models for the underlying, say

$$
\mathbb{M}=\{\mathcal{M}(\theta)\}_{\theta \in \Theta},
$$

such that, $P^{\theta}(h)$, the time 0 price of a claim with payoff function $h$ in the model $\mathcal{M}(\theta)$, is fairly easy to compute. If, in addition, for each $t$, the relation

$$
\theta \mapsto\left\{P^{\theta}(h)\right\}_{h \in \mathcal{D}_{t}},
$$

is invertible, we obtain a one-to-one correspondence between a set of admissible prices of $\mathcal{D}_{t}$ (each $\left\{P^{\theta}(h)\right\}_{h \in \mathcal{D}_{t}}$ is admissible by construction) and a (hopefully simple) space $\Theta$ :

$$
\left\{P^{\theta}(h)\right\}_{h \in \mathcal{D}_{t}} \mapsto \theta .
$$

We call the set $\Theta$ a code book and the above bijective correspondence a code-book relation, which allows us to think of the derivative prices from $\mathcal{D}_{t}$ in terms of a corresponding parameter $\theta \in \Theta$. In view of the above definitions, if at time $t$ there exists a value in the code-book $\theta_{t}$ which reproduces the market prices, then the model $\mathcal{M}\left(\theta_{t}\right)$ is tangent to the true model at time $t$.

Assuming that the set $\Theta$ is simple enough (an open subset of a linear space), we can now construct market models by putting in motion the value of the corresponding code-book $\theta_{t}$, and compute the resulting derivatives prices at any future time $t$ via (5). For the theoretical construction of market models that follows, we will have to assume that the initial (currently observed) set of derivatives prices can be represented by a model from a chosen class $\mathbb{M}$, and hence obtain $\theta_{0}$. However, in order to justify this assumption we need to choose the above family of simple models so that it is large enough and can in practice be calibrated to match at least approximately any feasible set of market prices.

It is worth mentioning that code-books, as general transforms for more convenient representation of derivatives prices, have been used by practitioners for a very long time: the examples include yield curve in the Treasury bond market, implied term structure of default probabilities for CDO tranches and implied volatility for the European options. However, here we require a specific form of construction for the code-book: this is dictated by our goal to model the prices of all derivatives simultaneously. As a result, the popular implied volatility surface is not a suitable code-book in the present setup.

We now define two important classes of tangent models and we review their main properties in the following two sections.

Examples of Sets of Derivatives. Coming back to models for equity trading, the following list is a sample of examples which can be found in papers we know, of and for which the above formalism applies:

- $\mathcal{D}_{t}=\left\{S, C_{t}(T, K) ; T>t\right\}$ for some fixed $K>0$ (case considered by Schoenbucher in [11])

- $\mathcal{D}_{t}=\left\{S, C_{t}(T, K) ; T>t\right\}$ for some fixed convex payoff function (case considered by Jacod and Protter in [7] and Schweizer and Wissel in [12]) 
- $\mathcal{D}_{t}=\left\{S, C_{t}(T, K) ; K>0\right\}$ for some fixed $T>t$ (case considered by Schweizer and Wissel in [13])

- $\mathcal{D}_{t}=\left\{S, C_{t}\left(T_{i}, K_{j}\right) ; i=1, \cdots, m, j=1, \cdots, n\right\}$ (case considered by Schweizer and Wissel in [?])

- $\mathcal{D}_{t}=\left\{S, C_{t}(T, K) ; T>t, K>0\right\}$ (case considered by Cont and Durrleman in [?] and Carmona and Nadtchiy in [?]).

For the rest of this paper we concentrate on the market given by the last example:

$$
\mathcal{D}_{t}=\left\{S, C_{t}(T, K) ; T>t, K>0\right\} .
$$

Notice that even though the set $\mathcal{D}_{t}$ is finite in practice, it is often assumed to be infinite (even of continuum power) in the financial mathematic and engineering literature.

Notice that due to the definition of a tangent model, we can identify such model with the law of the underlying process it produces, as opposed to the general case when a financial model is defined by the pair: "underlying process" and "market filtration". In the same way, by model $\mathcal{M}(\theta)$ we will understand a corresponding distribution of the underlying.

Tangent Diffusion Models. We say that a tangent model is a tangent diffusion model if it is given by a possibly inhomogeneous diffusion process. In other words, a model is a tangent diffusion model if it is tangent (in the sense of (4)) and consists of an underlying process $\tilde{S}$, which is a diffusion given by

$$
\tilde{S}_{t}=s+\int_{0}^{t} \tilde{S}_{u} \tilde{a}\left(u, \tilde{S}_{u}\right) d B_{u}
$$

with some initial condition $s$, local volatility function $\tilde{a}(.,$.$) and a Brownian motion B$.

The law of $\tilde{S}$ is then uniquely determined by $(s, \tilde{a}(.,)$.$) , where the surface \tilde{a}$ has to satisfy some regularity assumptions (see [?] and [?]). Clearly, the time 0 values of the underlying and the call price surface in any such model are given by $s$ and

$$
C^{s, \tilde{a}}(T, x)=\mathbb{E}\left(\tilde{S}_{T}-e^{x}\right)^{+}
$$

if we use the notation $K=e^{x}$ for the strike. From Dupire's formula (3), we can conclude that the above mapping from $(s, \tilde{a})$ to the couple ("value of the underlying", "prices of call options") is invertible, thus, producing a code-book.

If at a given moment of time $t$ there exists a value of the code-book $(s, \tilde{a})$, which reproduces the true market prices of all the call options and the underlying, then the model given by $(s, \tilde{a})$ is a tangent diffusion model at time $t$. In that case $s$ has to coincide with the current value of the underlying and $\tilde{a}$ is calibrated (fitted) to match the observed call prices.

Tangent Lévy Models. We say that a tangent model is a tangent Lévy model if is given by a possibly time-inhomogeneous Lévy (additive) process. To be more specific, a given model is a tangent Lévy model if it is tangent (in the sense of (4)) and consists of an underlying process $\tilde{S}$, which is a pure jump martingale given by

$$
\tilde{S}_{t}=s+\int_{0}^{t} \int_{\mathbb{R}} \tilde{S}_{u-}\left(e^{x}-1\right)[N(d x, d u)-\eta(d x, d u)],
$$


where $N(d x, d u)$ is a Poisson random measure associated with the jumps of $\log (\tilde{S})$, having an absolutely continuous deterministic compensator

$$
\eta(d x, d u)=\tilde{\kappa}(u, x) d x d u .
$$

The law of $\tilde{S}$ is then uniquely determined by $(s, \tilde{\kappa})$. Clearly, the time 0 values of the underlying and the call price surface in any such model are given by $s$ and

$$
C^{s, \tilde{\kappa}}(T, x)=\mathbb{E}\left(\tilde{S}_{T}-e^{x}\right)^{+}
$$

respectively. From the analytic representation of (9), provided in Section 4 (and discussed in more detail in [2]), it is not hard to see that the above mapping from $(s, \tilde{\kappa})$ to ("value of the underlying", "prices of the call options") is invertible, thus, producing a code-book.

As before, if at a given moment of time $t$ there exists a value of the code-book $(s, \tilde{\kappa})$, which reproduces the true market prices of call options and the underlying, then the model given by $(s, \tilde{\kappa})$ is a tangent Lévy model at time $t$.

2.1. Time-consistency of Calibration. It is important to remember that our standing assumption is that the prices of all contingent claims are given by conditional expectations in the true (unknown) model. Therefore, prescribing the dynamics of the code-book $\theta_{t}$ we have to make sure that the prices of derivatives produced by $\theta_{t}$ at each future time $t$ are indeed "the prices": they have to coincide with corresponding conditional expectations. In other words, the resulting model $\mathcal{M}\left(\theta_{t}\right)$ has to be tangent to the true model at each time $t$. This condition reflects the internal time-consistency of the dynamic calibration, and therefore, we further refer to it as the consistency of code-book dynamics (or simply "consistency"). If the dynamics of $\theta_{t}$ are consistent with a true model and $\mathcal{M}\left(\theta_{t}\right)$ is tangent to this true model at each time $t$, then we say that the true model and $\left(\theta_{t}\right)$ form a dynamic tangent model.

\section{Dynamic Tangent Diffusion Models}

In this approach, at each time $t$, the prices of all the call options are captured through the local volatility $\tilde{a}_{t}(.,$.$) defined with what is known as Dupire's formula, which we recalled earlier in the$ static case $t=0$ :

$$
\tilde{a}_{t}^{2}(T, K):=\frac{2 \frac{\partial}{\partial T} C_{t}(T, K)}{K^{2} \frac{\partial^{2}}{\partial K^{2}} C_{t}(T, K)} .
$$

As discussed above, this formula provides the code-book mapping from surfaces of call prices to local volatility functions. We switch to the new variables $\tau$ for the time-to-maturity and $x$ for the log-moneyness, writing

$$
h_{t}(\tau, x):=\log \tilde{a}_{t}^{2}\left(\tau, s e^{x}\right)
$$

for its logarithm. Using these notations, the analytic representation of the call prices produced by the static code-book $(s, \tilde{a})$

$$
c^{s, \tilde{a}}(\tau, x)=\frac{1}{s} C_{t}^{s, \tilde{a}}(t+\tau, \log s+x)
$$


takes the form of the Partial Differential Equation (PDE) for the normalized call prices

$$
\left\{\begin{array}{l}
\partial_{\tau} c^{s, \tilde{a}}(\tau, x)=e^{h(\tau, x)} D_{x} c^{s, \tilde{a}}(\tau, x), \tau>0, x \in \mathbb{R} \\
c^{s, \tilde{a}}(0, x)=\left(1-e^{x}\right)^{+} .
\end{array}\right.
$$

where we use the notation $D_{x}$ for the differential operator $D_{x}=\frac{1}{2}\left(\partial_{x^{2}}^{2}-\partial_{x}\right)$. Starting from a local volatility function $a$ (or equivalently its $\log$ arithm $h$ ) and ending with the solution of this PDE defines an operator $\mathbf{F}: h \mapsto c$ which plays a crucial role in the analysis of tangent diffusion models.

Once specific function spaces are chosen, formula (10) and the operator $\mathbf{F}$ provide a one-to-one correspondence between call option price surfaces and local volatility surfaces. This defines a codebook for the call prices. See for example [?] for more details.

Formal Definition of Dynamic Tangent Diffusion Models. As explained earlier, we assume that a pricing measure has been chosen (it does not have to be unique), and that under the probability structure it defines, the risk-neutral drift of the underlying index is zero as we ignore interest rate and dividend payments for the sake of simplicity.

We don't want to assume any specific model for the true underlying $S$, however, in order to describe market models explicitly we need to make some mild assumptions on $S$. When the filtration is Brownian, a natural specification is that the true underlying is a martingale of the form:

$$
d S_{t}=S_{t} \sigma_{t} d W_{t},
$$

for some adapted spot volatility process $\left(\sigma_{t}\right)$.

Now we need to specify dynamics of the code-book $\left(s_{t}, \tilde{a}_{t}\right)$. Notice that if we want these dynamics to be consistent (see the discussion in Subsection 2.1), we have to have $s_{t}=S_{t}$. Thus we define the code-book dynamics (time evolution) by

$$
\left\{\begin{array}{l}
s_{t}=S_{t}, \quad d S_{t}=S_{t} \sigma_{t} d B_{t}^{1}, \\
\tilde{a}_{t}(\tau, K)=\exp \left(\frac{1}{2} h_{t}\left(\tau, \log K / s_{t}\right)\right), \quad d h_{t}=\alpha_{t} d t+\sum_{n=1}^{m} \beta_{t}^{n} d B_{t}^{n},
\end{array}\right.
$$

where $B=\left(B^{1}, \ldots, B^{m}\right)$ is an $m$-dimensional Brownian motion ( $m$ could be $\infty$ ), the stochastic processes $\alpha$ and $\left\{\beta^{n}\right\}_{n=1}^{m}$ take values in spaces of functions of $\tau$ and $x$, and $\sigma$ is a scalar random process (see [?] and [?] for more details).

This type of model was first proposed by Derman and Kani in [6] and studied mathematically by Carmona and Nadtochiy in [?] and [?].

The main question we address is the following: "When is such a model consistent (i.e. a dynamic tangent model)?

Consistency of Dynamic Tangent Diffusion Models. The above question turns out to be equivalent to obtaining a necessary and sufficient conditions for all call prices produced by the code-book to be martingales.

Also, we would like to know, among all the parameters $\sigma, \alpha$, and $\beta$, which ones are constrained by the consistency and no-arbitrage conditions, and which ones can be chosen freely? The latter question is still open.

Starting from Itô's dynamics for $h$ (or equivalently $\tilde{a}$ ), an infinite dimensional version of Itô's formula shows that call prices are semi-martingales, and being able to compute their drifts should lead to no-arbitrage conditions merely stating that the call prices are martingales. Clearly, this reasoning 
depends upon proving that the mapping provided by the operator $\mathbf{F}$ is twice Fréchet differentiable. This strategy for the analysis of no-arbitrage was used in [1], whose main result we state below after we agree to denote by $p(h)$ the fundamental solution of the forward PDE

$$
\partial_{\tau} w(\tau, x)=e^{h(\tau, x)} D_{x} w(\tau, x)
$$

and by $q(h)$ the fundamental solution of the dual (backward) PDE

$$
\partial_{\tau} w(\tau, x)=-e^{h(\tau, x)} D_{x} w(\tau, x)
$$

It is proven in [1] that once the proper function spaces are chosen, the operator $\mathbf{F}$ is twice continuously Frechét-differentiable, and that for any $h, h^{\prime}, h^{\prime \prime} \in \mathcal{B}$, we have

$$
\mathbf{F}^{\prime}(h)\left[h^{\prime}\right]=\frac{1}{2} K\left[p(h), h^{\prime} e^{h}, q(h)\right],
$$

and

$$
\begin{aligned}
\mathbf{F}^{\prime \prime}(h)\left[h^{\prime}, h^{\prime \prime}\right]=\frac{1}{2}( & K\left[I\left[p(h), h^{\prime \prime} e^{h}, p(h)\right], h^{\prime} e^{h}, q(h)\right] \\
& \left.+K\left[p(h), h^{\prime} e^{h}, J\left[q(h), h^{\prime \prime} e^{h}, q(h)\right]\right]\right)
\end{aligned}
$$

where the operators $I, J$, and $K$ are defined by

- $I\left[\Gamma_{2}, f, \Gamma_{1}\right]\left(\tau_{2}, x_{2} ; \tau_{1}, x_{1}\right)$

$$
:=\int_{\tau_{1}}^{\tau_{2}} \int_{\mathbb{R}} \Gamma_{2}\left(\tau_{2}, x_{2} ; u, y\right) f(u, y) D_{y} \Gamma_{1}\left(u, y ; \tau_{1}, x_{1}\right) d y d u,
$$

- $J\left[\Gamma_{2}, f, \Gamma_{1}\right]\left(\tau_{2}, x_{2} ; \tau_{1}, x_{1}\right)$

$$
:=\int_{\tau_{1}}^{\tau_{2}} \int_{\mathbb{R}} D_{y} \Gamma_{2}\left(\tau_{2}, x_{2} ; u, y\right) f(u, y) \Gamma_{1}\left(u, y ; \tau_{1}, x_{1}\right) d y d u,
$$

- $K\left[\Gamma_{2}, f, \Gamma_{1}\right]\left(\tau_{2}, x_{2} ; \tau_{1}, x_{1}\right)$

$$
:=\int_{\tau_{1}}^{\tau_{2}} \int_{\mathbb{R}} \Gamma_{2}\left(\tau_{2}, x_{2} ; u, y\right) e^{y} f(u, y) \Gamma_{1}\left(u, y ; \tau_{1}, x_{1}\right) d y d u .
$$

Finally, as it is shown in [?], if we use the notations $\tilde{h}_{t}(T, x)=h_{t}\left(T-t, x-\log S_{t}\right)$, and $L(h):=$ $\log q(h)$, and provided that the processes $\sigma, \alpha$ and $\beta$ are chosen in appropriate spaces, then we have a dynamic tangent diffusion model if and only if the following two conditions are satisfied:

(1) Drift restriction:

$$
\tilde{\alpha}_{t}=\sigma_{t} \tilde{\beta}_{t}^{1}\left(\partial_{x} L\left(\tilde{h}_{t}\right)-L^{\prime}\left(\tilde{h}_{t}\right)\left[\partial_{x} \tilde{h}_{t}\right]\right)-\frac{1}{2} \sum_{n=1}^{\infty} \tilde{\beta}_{t}^{n}{ }^{2}-\sum_{n=1}^{\infty} \tilde{\beta}_{t}^{n} L^{\prime}\left(\tilde{h}_{t}\right)\left[\tilde{\beta}_{t}^{n}\right]
$$

(2) Spot volatility specification:

$$
\tilde{h}_{t}\left(t, S_{t}\right)=2 \log \sigma_{t}
$$


From the form of the above drift condition (14) and the spot volatility specification condition (15), it looks like $\beta$ is a free parameter whose choice completely determines both $\alpha$ and $\sigma$. However, the function $L(h)$ has a singularity at $\tau=0$ and some work is needed to identify a set of workable sufficient conditions on $\beta$ which guarantee that the drift condition (14) actually produces an admissible $\alpha_{t}$. This problem is still open.

Short Time-to-maturity Behavior of $L$. From classical results of S.Molchanov, S.Varadhan, I.Chavel and others, it follows that:

$$
L(h)(\tau, x)=-\frac{1}{2} \log \tau-\frac{\left(\int_{0}^{x} e^{-\frac{1}{2} h(0, y)} d y\right)^{2}}{2 \tau}+\hat{L}(h)(\tau, x),
$$

where $\hat{L}(h)(.,$.$) is a smooth function which satisfies a well posed initial-value problem (without$ singularities).
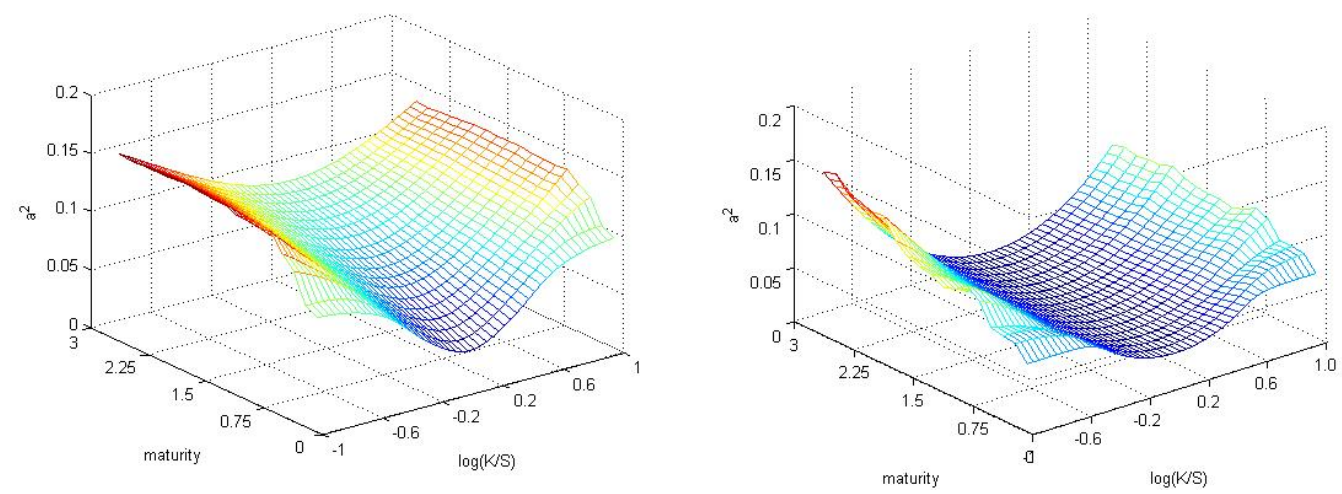

FIGURE 1. Local Volatility in Heston (left) and Hull-White (right) models.

One of the important questions which we address in this paper is "Given a set of call option prices, when can we use the local volatility as a (static) code book?" A classical result of Gyongyi [?] says that it is possible if the underlying index $S_{t}$ is an Itô process satisfying some mild regularity conditions.

\section{Dynamic Tangent Lévy Models}

Our work [2] on tangent Lévy models was a natural attempt to relax the assumption that $S_{t}$ is an Itô's process, and introduce jumps in its dynamics, the relevant question being: "What is the right substitute for the local volatility code?"

Using processes with jumps in financial modeling goes back to the 1976 Merton's pioneering work [?], and fitting option prices with Lévy-based models has also a long history. At the risk of missing important contributions, we mention for example the series of works by Carr, Geman, Madan, Yor, and Seneta between 1990 and 2005 [9, 5, 3] on models with jumps of infinite activity, such as the Variance Gamma (VG) and CGMY models, and the easy to use double exponential model of Kou [8]. Still in the static case at time $t=0$, P.Carr, H.Geman, D.Madan and M.Yor noticed in their 
2004 paper [4] that Dupire's local volatility can be interpreted as an $S_{t}$ dependent time change and proposed to use a Markovian time change of a Lévy process to introduce what they called Local Lévy models. However, following this approach to define tangent Lévy models would lead to the same level of complexity in the formulation of the consistency conditions as in the case of tangent diffusion models. For this reason, we chose to define the tangent Lévy models in a different way - see the definition in Subsection 2.

Recall (9) - the expression for call prices in pure jump exponential additive models. This formula, together with the specification of " $s$ " as the current value of the underlying, establishes the codebook relation, and, as it was stated above, in order to construct a dynamic tangent model we need to prescribe dynamics of the code-book $(s, \tilde{\kappa})$ and make sure they are consistent. However, in order to study consistency of the code-book dynamics, we need to have a convenient analytic representation of the code-book mapping. Therefore, we make use of the following PIDE representation of the call prices produced by $\tilde{\kappa}$ and $s$ via (9)

$$
\left\{\begin{array}{c}
\partial_{T} C^{s, \tilde{\kappa}}(T, x)=\int_{\mathbb{R}} \psi(\tilde{\kappa}(T, \cdot) ; x-y) D_{y} C^{s, \tilde{\kappa}}(T, y) d y \\
\left.C^{s, \tilde{\kappa}}(T, x)\right|_{T=0}=\left(s-e^{x}\right)^{+}
\end{array}\right.
$$

where we recall the notation $C^{s, \tilde{\kappa}}(T, x)$ for the price of call option with maturity $T$ and strike $e^{x}$ produced by $(s, \tilde{\kappa})$ via (9). We also introduce $D_{x}:=\partial_{x^{2}}^{2}-\partial_{x}$ and the double exponential tail function $\psi$, defined by

$$
\psi(f, x)= \begin{cases}\int_{-\infty}^{x}\left(e^{x}-e^{z}\right) f(z) d z & x<0 \\ \int_{x}^{\infty}\left(e^{z}-e^{x}\right) f(z) d z & x>0 .\end{cases}
$$

Clearly, the presence of convolutions and constant coefficient differential operators in (17) are screaming for the use of Fourier transform.

4.1. Fourier Transform. Unfortunately, the setup is not Fourier transform friendly as the initial condition in (17) is not an integrable function! In order to overcome this difficulty, we work with derivatives. Before taking Fourier transform (we use a "hat" for functions in Fourier space), we differentiate both sides of the PIDE in (17) introducing the notation $\Delta^{s, \tilde{\kappa}}(T, x)=-\partial_{x} C^{s, \tilde{\kappa}}(T, x)$. Thus, we obtain

$$
\left\{\begin{array}{c}
\partial_{T} \hat{\Delta}^{s, \tilde{\kappa}}(T, \xi)=-\left(4 \pi^{2} \xi^{2}+2 \pi i \xi\right) \hat{\psi}(\tilde{\kappa}(T, \cdot), \xi) \hat{\Delta}^{s, \tilde{\kappa}}(T, \xi) \\
\left.\hat{\Delta}^{s, \tilde{\kappa}}(T, \xi)\right|_{T=0}=\frac{\exp \{\log s(1-2 \pi i \xi)\}}{1-2 \pi i \xi}
\end{array}\right.
$$

The above equation, in particular, gives us a mapping: $C^{s, \tilde{\kappa}} \rightarrow \hat{\Delta}^{s, \tilde{\kappa}} \rightarrow \hat{\psi} \rightarrow \tilde{\kappa}$.

One sees that in order to go from $\tilde{\kappa}$ and $s$ to call prices we only need to solve the simple evolution equation in Fourier domain (19), and obtain $\hat{\Delta}^{s, \tilde{\kappa}}$ in closed form. We can recover $\Delta^{s, \tilde{\kappa}}(T, x)=$ 
$-\partial_{x} C^{s, \tilde{\kappa}}(T, x)$ by inverting the Fourier transform, and a plain integration gives

$$
\begin{aligned}
& C^{s, \tilde{\kappa}}(T, x)=s \lim _{\lambda \rightarrow+\infty} \int_{\mathbb{R}} \frac{e^{2 \pi i \xi \lambda}-e^{2 \pi i \xi(x-\log s)}}{2 \pi i \xi(1-2 \pi i \xi)} \\
& \quad \exp \left(-2 \pi\left(2 \pi \xi^{2}+i \xi\right) \int_{t \wedge T}^{T} \hat{\psi}(\tilde{\kappa}(u, \cdot), \xi) d u\right) d \xi
\end{aligned}
$$

providing the mapping: $\tilde{\kappa} \rightarrow \hat{\psi} \rightarrow C^{s, \tilde{\kappa}}$.

Formal Definition of Dynamic Tangent Lévy Models and Consistency Results. As in the case of the tangent diffusion models we need to put the static code-book $(s, \tilde{\kappa})$ in motion, and construct a pair of stochastic processes $\left(s_{t}, \tilde{\kappa}_{t}\right)_{t \in[0, \bar{T}]}$ under the pricing measure. Clearly, we would like these processes to be consistent with the true model. As before, we would like to keep the true model for the underlying as general as possible. However, in order to make the results more specific, we need to make some assumptions on the dynamics of the underlying. In particular, we assume that the underlying $S$ is a positive pure jump martingale under the pricing measure: more precisely, we assume that it is given by

$$
S_{t}=S_{0}+\int_{0}^{t} \int_{\mathbb{R}} S_{u-}\left(e^{x}-1\right)\left(M(d x, d u)-K_{u}(x) d x d u\right)
$$

for some (unknown) integer valued random measure $M$ whose predictable compensator is absolutely continuous, i.e. of the form $K_{\omega, u}(x) d x d u$, and such that almost surely in $\omega \in \Omega, K_{\omega, u}$ is in the Banach space $\mathcal{B}^{0}$ constructed in Section 3 of [?].

It may seem too restrictive to assume that the underlying process has no continuous martingale component and that the compensator of $M$ is absolutely continuous. These assumptions are dictated by our choice of the code-book, which is based on pure jump processes without fixed points of discontinuity. To see this, recall, for example, that the short-maturity properties of call prices produced by pure jump models are incompatible with the presence of a continuous component in the underlying dynamics. The next section provides an extension of the present code-book and, as a result, allows for slightly more general dynamics of the underlying.

Let us now specify the dynamics of $\left(s_{t}, \tilde{\kappa}_{t}\right)$. As it was mentioned above, if we want the model corresponding to $\left(s_{t}, \tilde{\kappa}_{t}\right)$ to be tangent to the true model at time $t$ (in other words, if we want $\left(s_{t}, \tilde{\kappa}_{t}\right)$ to be the value of a code that reproduces the market prices at time $t$ ), then $s_{t}$ has to coincide with $S_{t}$, and its dynamics be given by (20). Therefore, the only additional process whose time-evolution we need to specify is $\left(\tilde{\kappa}_{t}\right)_{t \in[0, \bar{T}]}$. For the sake of convenience, we will model $\tilde{\kappa}_{t}$ in the form

$$
\tilde{\kappa}_{t}(\tau, x)=\kappa_{t}(t+\tau, x),
$$

where

$$
\kappa_{t}=\kappa_{0}+\int_{0}^{t} \alpha_{u} d u+\sum_{n=1}^{m} \int_{0}^{t} \beta_{u}^{n} d B_{u}^{n}
$$

where $B=\left(B^{1}, \ldots, B^{m}\right)$ is an $m$-dimensional Brownian motion ( $m$ can be $\left.\infty\right), \alpha$ is a progressively measurable integrable stochastic process with values in a Banach space $\mathcal{B}$, and $\beta=\left(\beta^{1}, \ldots, \beta^{m}\right)$ is a vector of progressively measurable square integrable stochastic processes taking values in a Hilbert space $\mathcal{H}$. Please see Section 3 of [?] for the precise definitions of the spaces $\mathcal{B}$ and $\mathcal{H}$. 
Notice again, that the dynamics of $\kappa_{t}$ could, in principle, include jumps. We, however, restrict our framework to the continuous evolution of $\kappa$ in order to keep the results and their derivations more transparent.

Thus, a dynamic tangent Lévy model is defined by the pair of equations (20) and (21), given that such dynamics are consistent, or in other words, given that for any $(T, x) \in(0, \bar{T}] \times \mathbb{R}$ the following equality is satisfied almost surely for all $t \in[0, T)$

$$
C^{S_{t}, \tilde{\kappa}_{t}}(T, x)=\mathbb{E}\left(\left(S_{T}-K\right)^{+} \mid \mathcal{F}_{t}\right)
$$

This form of the consistency condition is, of course, not very convenient. It is important to characterize the consistency of the code-book dynamics ((20) and (21)) explicitly in terms of the input parameters: $\alpha, \beta$ and $K$. Such explicit formulation of the consistency condition is one of the main results of [?], and it is given in Theorem 1 and Corollary 4 of the above mentioned paper. In order to state this result we introduce the notation:

$$
\bar{\beta}_{t}^{n}(T, x):=\int_{t \wedge T}^{T} \beta_{t}^{n}(u, x) d u, \quad \Psi(f ; x)=-e^{x} \int_{x}^{\operatorname{sign}(x) \infty} f(y) d y .
$$

Assuming $S$ is a true martingale, $\kappa \geq 0$ and $\beta$ satisfies the alternative regularity assumptions ARA1-ARA3 given in Section 3 of [?], the dynamic tangent Lévy model given by (20) and (21) is consistent if and only if the following conditions are satisfied:

(1) Drift restriction:

$$
\begin{gathered}
\alpha_{t}(T, x)=-e^{-x} \sum_{n=1}^{m} \int_{\mathbb{R}} \partial_{y^{2}}^{2} \Psi\left(\bar{\beta}_{t}^{n}(T) ; y\right)\left[\Psi\left(\beta_{t}^{n}(T) ; x-y\right)-\left(1-y \partial_{x}\right) \Psi\left(\beta_{t}^{n}(T) ; x\right)\right] \\
-\Psi\left(\bar{\beta}_{t}^{n}(T) ; y\right) \Psi\left(\beta_{t}^{n}(T) ; x-y\right) d y
\end{gathered}
$$

(2) Compensator specification: $K_{t}(x)=\kappa_{t}(t, x)$.

Model Specification and Existence Result. Denoting by $\rho$ the weight function

$$
\rho(x):=e^{-\lambda|x|}\left(|x|^{-1-\delta} \vee 1\right),
$$

with some $\lambda>1$ and $\delta \in(0,1)$, and switching from $\kappa_{t}$ to $\check{\kappa}_{t}$ given by $\check{\kappa}_{t}(T, x)=\kappa_{t}(T, x) / \rho(x)$, we can easily force $\check{\kappa}_{t}$ to take values in a more convenient space of continuous functions, in which its maximal and minimal values can be controlled. Introducing the weighted drift $\check{\alpha}_{t}=\alpha_{t} / \rho$, weighted diffusion terms $\left\{\check{\beta}_{t}^{n}=\beta_{t}^{n} / \rho\right\}_{n=1}^{m}$ (which take values in corresponding function spaces, see Section 5 of [?] for details) and the stopping time

$$
\tau_{0}=\inf \left\{t \geq 0: \inf _{T \in[t, \bar{T}], x \in \mathbb{R}} \check{\kappa}_{t}(T, x) \leq 0\right\},
$$

( $\tau_{0}$ is predictable and $\check{\kappa}_{t \wedge \tau_{0}}$ is nonnegative), we can specify the model as follows:

- Assume that the market filtration supports a Brownian motion $\left\{B^{n}\right\}_{n=1}^{m}$ and an independent Poisson random measure $N$ with compensator $\rho(x) d x d t$.

- Denote by $\left\{\left(t_{n}, x_{n}\right)\right\}_{n=1}^{\infty}$ the atoms of $N$. Then $M$ can be defined by its atoms

$$
\left\{\left(t_{n}, W\left[\check{\kappa}_{t_{n}}\left(t_{n}, .\right)\right]\left(x_{n}\right)\right)\right\}_{n=1}^{\infty},
$$


for some deterministic mapping $f(.) \mapsto W[f]($.$) , so that it has the desired compensator$ $\rho(x) \check{\kappa}_{t}(t, x) d x d t$, and therefore, the compensator specification is satisfied. An explicit expression for the mapping $W$ is given in Section 5 of [?].

- Rewrite the right hand side of the drift restriction using $\check{\beta}$ instead of $\beta$, and denote the resulting quadratic operator by $Q^{\check{\beta}_{t}}(T, x)$. Construct $\check{\kappa}_{t}$ by integrating " $\check{\beta}_{t} \cdot d B_{t}+Q^{\check{\beta}_{t}} d t$ ", and stop it at $\tau_{0}$. Such $\breve{\kappa}$ will satisfy the drift restriction and the nonnegativity property.

In addition, $\beta_{t}=\rho \check{\beta}_{t}$ satisfies the alternative regularity assumptions due to the choice of state space for $\check{\beta}_{t}$.

- If, in addition, we assume that $\check{\beta}$ is independent of $N$, we can guarantee that $S$, produced by (20) and the above choice of $M$, is a true martingale. Thus, the above specification allows to determine the model uniquely through $N, B$ and $\breve{\beta}$.

As a result we obtain the following class of code-book dynamics:

$$
\left\{\begin{array}{c}
S_{t}=S_{0}+\int_{0}^{t} \int_{\mathbb{R}} S_{u-}\left(\exp \left(W\left[\check{\kappa}_{u}(u, .)\right](x)\right)-1\right)(N(d x, d u)-\rho(x) d x d u), \\
\tilde{\kappa}_{t}(T, x)=\rho(x) \check{\kappa}_{t}(t+T, x), \quad \check{\kappa}_{t}=\check{\kappa}_{0}+\int_{0}^{t} Q^{\check{\beta}_{u}} \mathbf{1}_{u \leq \tau_{0}} d u+\sum_{n=1}^{m} \int_{0}^{t} \check{\beta}_{u}^{n} \mathbf{1}_{u \leq \tau_{0}} d B_{u}^{n}
\end{array}\right.
$$

Theorem 2 in [?] states that for any square integrable stochastic process $\check{\beta}$ the above system has a unique solution, and if, in addition, $\check{\beta}$ is independent of $N$, then the resulting processes $\left(S_{t}\right)_{t \in[0, \bar{T}]}$ and $\left(\tilde{\kappa}_{t}\right)_{t \in[0, \bar{T}]}$ are consistent, and, therefore, form a dynamic tangent Lévy model.

This "local existence" result, albeit limited (the presence of stopping time $\tau_{0}$ and the independence assumption should eventually be relaxed, as it is demonstrated by the example that follows), provides a method for construction of the future evolution of the market code-book, starting from any given one. In practice, this means that, if we are able to calibrate a model from the chosen space of pure jump exponential additive models to the currently observed option prices, we can use the above result to generate a large family of dynamic stochastic models for the future joint evolution of the option prices (or, equivalently, the implied volatility surface) and the underlying.

4.2. Example of Dynamic Tangent Lévy Model. The following tangent Lévy model was proposed in [2]. Its analysis and implementation on real market data is being carried out in [10]. Here we outline the main steps of the analysis to illustrate the versatility of the model, and the fact that it does provide an answer to the nagging question of the Monte Carlo simulation of arbitrage free time evolutions of implied volatility surfaces.

- Choose $m=1$, and $\check{\beta}_{t}(T, x)=\gamma_{t} C(x)$,

- Let $\gamma_{t}=\frac{\sigma}{\epsilon}\left(\inf _{T \in[t, \bar{T}], x \in \mathbb{R}} \check{\kappa}_{t}(T, x) \wedge \epsilon\right)$, for some $\sigma, \epsilon>0$,

- and $C(x)=e^{-\lambda^{\prime}|x|}(|x| \wedge 1)^{\delta} \tilde{C}(x)$, for some $\lambda^{\prime}>0, \delta \in(0,1)$ and some absolutely continuous function $\tilde{C}$ with bounded and absolutely integrable derivative.

- Then

$$
\check{\kappa}_{t}(T, x)=\check{\kappa}_{0}(T, x)+\frac{T-t}{2} A(x) \int_{0}^{t} \gamma_{u}^{2} d u+C(x) \int_{0}^{t} \gamma_{u} d B_{u}
$$



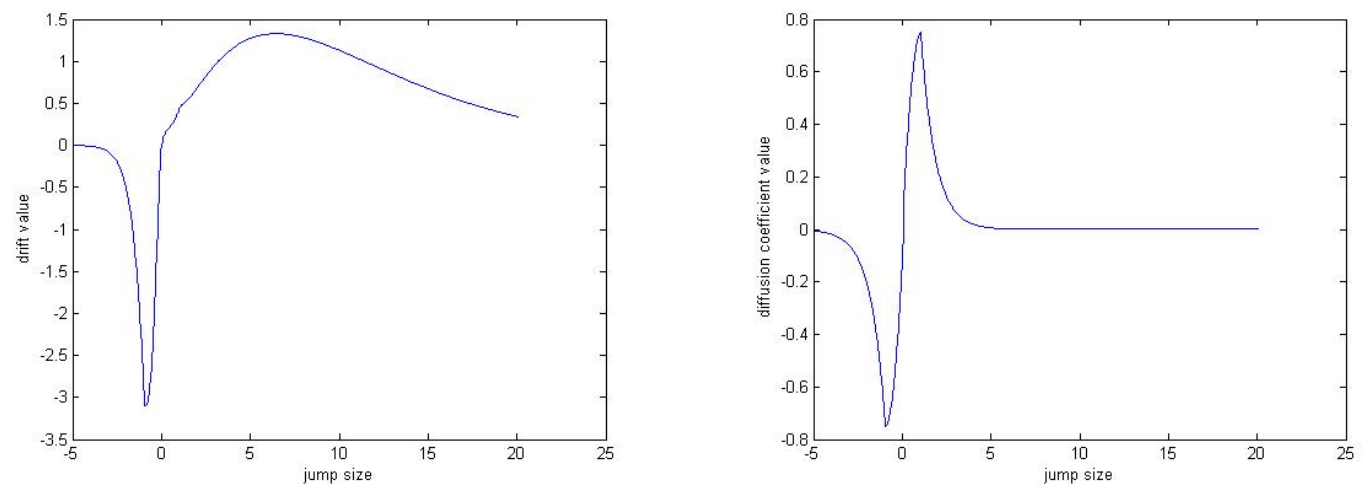

Figure 2. Example of Tangent Lévy Model: Drift (left) and diffusion coefficient (right) of $\tilde{\kappa}$, as functions of jump size $x$.
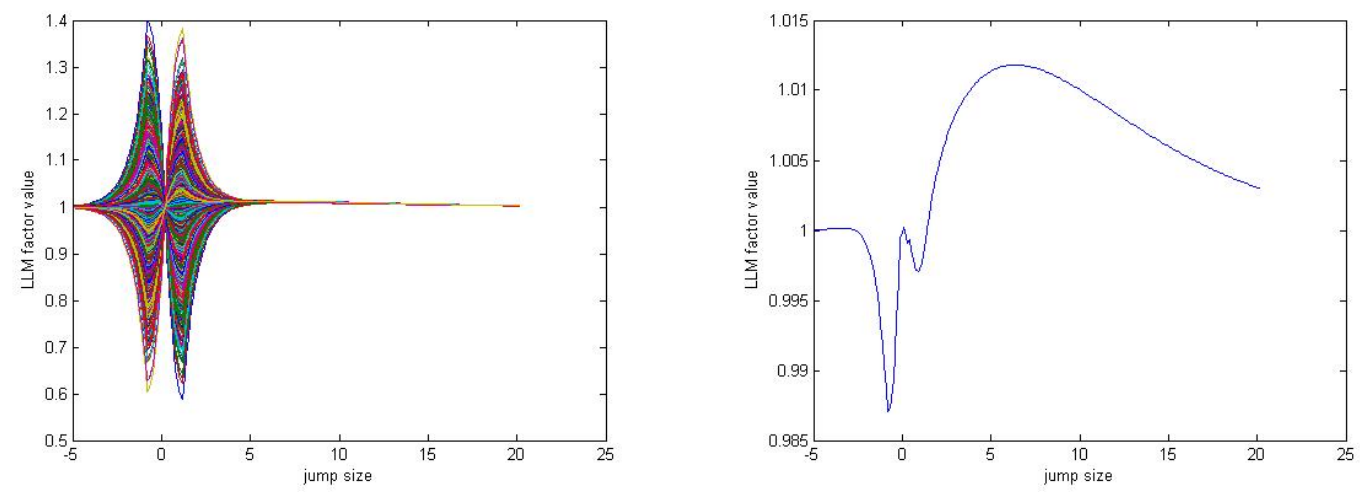

FIGURE 3. Simulated values of $\tilde{\kappa}_{T}(T,$.$) (left) and their average (right).$

where $A$ is obtained from $C$ via the "drift restriction".

It is worth mentioning that, as shown in Proposition 4 of [?], the process $\check{\kappa}$ defined in this way always stays positive.

\section{Extension of Dynamic Tangent Lévy Models}

Notice that the dynamic tangent Lévy models introduced above do not allow for a continuous martingale component in the evolution of the underlying. This is a direct consequence of our choice of the space of tangent models: they assume a pure jump evolution of the underlying and this implies pure jump dynamics in the true model for the underlying, since certain asymptotic properties of the marginal distributions of pure jump processes are incompatible with the presence of continuous martingale component (recall the "explosion" of Dupire's local volatility in models with jumps). Thus, in this section we consider an extension of the space of tangent Lévy models introduced above, 
which, although can be described using the same techniques, includes underlying processes with nontrivial continuous martingale components.

Recall the definition of the space of tangent Lévy models and consider its extension in the sense that we allow the underlying in each of the tangent model to be given by

$$
\tilde{S}_{t}=s+\int_{0}^{t} \tilde{\Sigma}(u) d \tilde{B}_{u}+\int_{0}^{t} \int_{\mathbb{R}} \tilde{S}_{u-}\left(e^{x}-1\right)[N(d x, d u)-\tilde{\kappa}(u, x) d x d u]
$$

for a one-dimensional Brownian motion $\tilde{B}$ independent of the Poisson random measure $N$ with compensator $\tilde{\kappa}(u, x) d x d u$. The class of such models is then parameterized by $(s, \tilde{\Sigma}(),. \tilde{\kappa}(.,)$.$) . As$ before, we introduce the call prices produced by $(s, \tilde{\Sigma}, \tilde{\kappa})$

$$
C^{s, \tilde{\Sigma}, \tilde{\kappa}}(T, x)=\mathbb{E}\left(\tilde{S}_{T}-e^{x}\right)^{+}
$$

and derive their analytic representation.

Finally, we put the static code-book in motion, producing the following dynamics of the code-book:

$$
\left\{\begin{array}{c}
s_{t}=S_{t}, \quad S_{t}=S_{0}+\int_{0}^{t} S_{u} \sigma_{u} d B_{u}^{1}+\int_{0}^{t} \int_{\mathbb{R}} S_{u-}\left(e^{x}-1\right)\left(M(d x, d u)-K_{u}(x) d x d u\right), \\
\tilde{\kappa}_{t}(T, x)=\kappa_{t}(t+T, x), \quad \kappa_{t}=\kappa_{0}+\int_{0}^{t} \alpha_{u} d u+\sum_{n=1}^{m} \int_{0}^{t} \beta_{u}^{n} d B_{u}^{n}, \\
\tilde{\Sigma}_{t}(T)=\Sigma_{t}(t+T), \quad \Sigma_{t}=\Sigma_{0}+\int_{0}^{t} \mu_{u} d u+\sum_{n=1}^{m} \int_{0}^{t} \nu_{u}^{n} d B_{u}^{n},
\end{array}\right.
$$

where $B=\left(B^{1}, \ldots, B^{m}\right)$ is a multidimensional Brownian motion, $M$ is an integer valued random measure with predictable compensator $K_{u}(\omega, x) d x d u, \alpha$ and $\beta^{n}$ are progressively measurable stochastic processes satisfying natural integrability properties and taking values in $\mathcal{B}$ and $\mathcal{H}$ respectively (see Section 3 of [?] for definitions). The progressively measurable integrable stochastic processes $\mu$ and $\nu^{n}$ take values, respectively, in the space of continuous functions on $[0, \bar{T}]$, equipped with "sup" norm, and in the Hilbert space of absolutely continuous functions on $[0, \bar{T}]$ with square integrable derivatives. The following result analyzes the consistency of the above dynamics.

Theorem 1. Assume that $\left(S_{t}\right)_{t \in[0, \bar{T}]}$ is a martingale, $\beta$ satisfies the alternative regularity assumptions ARA1-ARA2 in Section 3 of [?] and $\kappa_{t}(T, x) \geq 0$, almost surely for all $t \in[0, \bar{T})$ and almost all $(T, x) \in[t, \bar{T}] \times \mathbb{R}$. Then processes $\left(S_{t}, \Sigma_{t}, \kappa_{t}\right)_{t \in[0, \bar{T}]}$ satisfying (25) are consistent (and thus, form an extended dynamic tangent Lévy model) if and only if the following conditions hold almost surely for almost every $x \in \mathbb{R}$ and $t \in[0, \bar{T})$, and all $T \in(t, \bar{T}]$ :

(1) Drift restriction:

$$
\begin{array}{r}
\alpha_{t}(T, x)=-e^{-x} \sum_{n=1}^{m} \int_{\mathbb{R}} \partial_{y^{2}}^{2} \Psi\left(\bar{\beta}_{t}^{n}(T) ; y\right)\left[\Psi\left(\beta_{t}^{n}(T) ; x-y\right)-\left(1-y \partial_{x}\right) \Psi\left(\beta_{t}^{n}(T) ; x\right)\right] \\
-\Psi\left(\bar{\beta}_{t}^{n}(T) ; y\right) \Psi\left(\beta_{t}^{n}(T) ; x-y\right) d y-\sigma_{t}\left(\partial_{x}+2\right) \beta_{t}^{1}(T, x),
\end{array}
$$

(2) Compensator specification: $K_{t}(x)=\kappa_{t}(t, x)$,

(3) Volatility specification: $\sigma_{t}^{2}=\Sigma_{t}^{2}(t)$,

(4) Stability of volatility: $\mu \equiv 0, \nu \equiv 0$. 
Proof:

We first recall Proposition 1 from Section 4 of [?], which states that the dynamics of the codebook are consistent if and only if the call prices $\left(C^{S_{t}, \tilde{\Sigma}_{t}, \tilde{\kappa}_{t}}(T, x)\right)_{t \in[0, \bar{T})}$ produced by the code-book are martingales. It is not hard to see, by essentially repeating the proof of the proposition, that its statement holds in the present setup.

Thus, we need to characterize the martingale property of call prices produced by dynamic codebook in terms of the input parameters of the model. This, again, can be done along the lines presented in Section 4 of [?]. Recall that the analytic representation of the call prices produced by the code-book is obtained in the Fourier domain first (see (19) above). Thus, analogous to the case of tangent Lévy models, we introduce $\Delta^{s, \tilde{\Sigma}, \tilde{\kappa}}(T, x)=-\partial_{x} C^{s, \tilde{\Sigma}, \tilde{\kappa}}(T, x)$, and $\hat{\Delta}^{s, \tilde{\Sigma}, \tilde{\kappa}}(T, \xi)$ as the Fourier transform of $\Delta^{s, \tilde{\Sigma}, \tilde{\kappa}}(T,$.$) . Then we can rewrite (19) in the present setup (with one additional term on the right$ hand side of the equation) and obtain

$$
\hat{\Delta}^{S_{t}, \tilde{\Sigma}_{t}, \tilde{\kappa}_{t}}(T, \xi)=\frac{e^{\log S_{t}(1-2 \pi i \xi)}}{1-2 \pi i \xi} \exp \left(-2 \pi\left(2 \pi \xi^{2}+i \xi\right) \int_{T \wedge t}^{T} \frac{1}{2} \Sigma_{t}^{2}(u)+\hat{\psi}\left(\kappa_{t}(u, \cdot) ; \xi\right) d u\right)
$$

where $\hat{\psi}$ is the Fourier transform of $\psi$ defined in (18).

It is not hard to repeat the proof of Theorem 1 in Section 4 of [?] to see that the martingale property of $C^{S_{t}, \tilde{\Sigma}_{t}, \tilde{\kappa}_{t}}$ is, in fact, equivalent to the martingale property (in fact, even a local martingale property) of $\hat{\Delta}^{S_{t}, \tilde{\Sigma}_{t}, \tilde{\kappa}_{t}}$. Then, applying the Ito's rule to (26) (repeat the proofs of Propositions 1 and 2 and their corollaries from Section 4 of [?] for the rigorous derivation of this), we obtain

$$
\begin{aligned}
& d\left(\hat{\Delta}^{S_{t}, \tilde{\Sigma}_{t}, \tilde{\kappa}_{t}}(T, \xi)\right)=\hat{\Delta}^{S_{t}, \tilde{\Sigma}_{t}, \tilde{\kappa}_{t}}(T, \xi) 2 \pi i \xi(1-2 \pi i \xi)\left[\hat{\psi}\left(\kappa_{t}(t, .)-K_{t}(.) ; \xi\right)+\frac{1}{2}\left(\Sigma_{t}^{2}(t)-\sigma_{t}^{2}\right)\right. \\
& +\pi i \xi(1-2 \pi i \xi) \sum_{n=1}^{m}\left(\int_{t \wedge T}^{T} \Sigma_{t}(u) \nu_{t}^{n}(u) d u\right)^{2}-\int_{t \wedge T}^{T} \Sigma_{t}(u) \mu_{t}(u)+\frac{1}{2}\left|\nu_{t}(u)\right|^{2} d u \\
& +\pi i \xi(1-2 \pi i \xi) \sum_{n=1}^{m}\left(\int_{t \wedge T}^{T} \hat{\psi}\left(\beta_{t}^{n}(u, .) ; \xi\right) d u\right)^{2}-\int_{t \wedge T}^{T} \hat{\psi}\left(\alpha_{t}(u, .) ; \xi\right) d u \\
& \left.-(1-2 \pi i \xi) \sigma_{t} \int_{t \wedge T}^{T} \Sigma_{t}(u) \nu_{t}^{1}(u)+\hat{\psi}\left(\beta_{t}^{1}(u, .) ; \xi\right) d u\right] d t+[. .] d Z_{t}
\end{aligned}
$$

where $Z$ is a local martingale.

Denote the drift of the above equation by $\Gamma_{t}(T, \xi)$. We need to find necessary and sufficient conditions for $\Gamma_{t}(T, \xi) / \hat{\Delta}^{S_{t}, \tilde{\Sigma}_{t}, \tilde{\kappa}_{t}}(T, \xi)$ to be zero, for all $T \in(t, \bar{T})$ and $\xi \in \mathbb{R}$. Since this expression is absolutely continuous as a function of $T \in(t, \bar{T})$, it vanishes if and only if its value at $T=t$ is zero and the values of its derivative are zero for all $(T, \xi) \in(t, \bar{T}) \times \mathbb{R}$. Thus, we obtain a system of two equations: 


$$
\begin{aligned}
& \hat{\psi}\left(\kappa_{t}(t, \cdot)-K_{t}(.) ; \xi\right)+\frac{1}{2}\left(\Sigma_{t}^{2}(t)-\sigma_{t}^{2}\right)=0 \\
& 2 \pi i \xi(1-2 \pi i \xi) \sum_{n=1}^{m} \Sigma_{t}(T) \nu_{t}^{n}(T) \int_{t \wedge T}^{T} \Sigma_{t}(u) \nu_{t}^{n}(u) d u-\Sigma_{t}(T) \mu_{t}(T)+\frac{1}{2}\left|\nu_{t}(T)\right|^{2} \\
& \quad+2 \pi i \xi(1-2 \pi i \xi) \sum_{n=1}^{m} \hat{\psi}\left(\beta_{t}^{n}(u, \cdot) ; \xi\right) \int_{t \wedge T}^{T} \hat{\psi}\left(\beta_{t}^{n}(u, \cdot) ; \xi\right) d u-\hat{\psi}\left(\alpha_{t}(T, \cdot,) ; \xi\right) \\
& \quad-(1-2 \pi i \xi) \sigma_{t}\left(\Sigma_{t}(T) \nu_{t}^{1}(T)+\hat{\psi}\left(\beta_{t}^{1}(T, \cdot) ; \xi\right)\right)=0
\end{aligned}
$$

Now, recall that Fourier transform of an absolutely integrable function converges to zero as the argument goes to infinity. Also notice that multiplication by " $-2 \pi i \xi$ " in the Fourier domain corresponds to taking derivative in the original domain. Due to the alternative regularity assumptions, $\partial_{x} \psi\left(\beta_{t}^{n}(T,) ; x.\right)=\Psi\left(\beta_{t}^{n}(T,) ; x.\right)$ is absolutely integrable in $x \in \mathbb{R}$, therefore, $\xi \hat{\psi}\left(\beta_{t}^{n}(T,.) ; \xi\right) \rightarrow 0$, as $|\xi| \rightarrow \infty$. Using this observation, we can split the above system into the following parts

$$
\begin{aligned}
& \kappa_{t}(t, x)-K_{t}(x)=0, \quad \Sigma_{t}^{2}(t)-\sigma_{t}^{2}=0, \\
& \sum_{n=1}^{m} \Sigma_{t}(T) \nu_{t}^{n}(T) \int_{t \wedge T}^{T} \Sigma_{t}(u) \nu_{t}^{n}(u) d u=0, \quad \mu_{t}(T)=0, \\
& 2 \pi i \xi(1-2 \pi i \xi) \sum_{n=1}^{m} \hat{\psi}\left(\beta_{t}^{n}(u, \cdot) ; \xi\right) \int_{t \wedge T}^{T} \hat{\psi}\left(\beta_{t}^{n}(u, \cdot) ; \xi\right) d u \\
& \quad-(1-2 \pi i \xi) \sigma_{t} \hat{\psi}\left(\beta_{t}^{1}(T, \cdot) ; \xi\right)-\hat{\psi}\left(\alpha_{t}(T, \cdot) ; \xi\right)=0,
\end{aligned}
$$

which, after inverting the Fourier transform and operator $\psi$ (see the end of the proof of Theorem 1 in Section 4 of [?]), yields the statement of the theorem.

As one can see, the parameter $\Sigma_{t}$ in the above tangent models cannot change as a continuous stochastic process in $t$, and therefore, the spot volatility $\sigma_{t}$ has to be deterministic. This surprising result can be interpreted as follows: calibrating exponential additive model to the call option market at each time, assuming that the parameters of the calibration change continuously, one has to keep the same continuous quadratic variation component $\Sigma^{2}($.$) in order to avoid arbitrage.$

\section{CONCLuSions}

In this paper we described two classes of tangent (consistent) models when the call price surface can be coded by:

- a Local Volatility surface

- a Tangent Lévy Density.

Each class corresponds to a different type of dynamics of the underlying, continuous in the first case and pure jump in the second, while keeping the semimartingale property. Our description of tangent Lévy models is complete in the sense that for any admissible value of the free parameter in a given linear space, we can construct a unique arbitrage-free market model. Finally, the last contribution of 
this paper is to generalize the class of tangent Lévy models to include underlying processes with a diffusive component, and to extend the characterization of the consistency of the model (including the classical drift condition) to this enlarged class of models going beyond the pure jump underlying models studied in [?].

\section{REFERENCES}

[1] R. Carmona and S. Nadtochiy. An infinite dimensional stochastic analysis approach to local volatility dynamic models. Communications on Stochastic Analysis, 2(1):109 - 123, 2008.

[2] R. Carmona and S. Nadtochiy. Tangent Lévy models. Finance and Stochastics, 14(?):??-??, 2010.

[3] P. Carr, H. Geman, D. Madan, and M. Yor. The fine structure of asset returns: An empirical investigation. Journal of Business, 75(2):305 - 332, 2002.

[4] P. Carr, H. Geman, D. Madan, and M. Yor. From local volatility to local Lévy models. Quantitative Finance, 4:581 $588,2005$.

[5] P. Carr and D. Madan. Option valuation using the fast Fourier transform. Journal of Computational Finance, 2(5):61 $-73,1998$

[6] E. Derman and I. Kani. Stochastic implied trees: Arbitrage pricing with stochastic term and strike structure of volatility. International Journal of Theoretical and Applied Finance, 1:61-110, 1998.

[7] J. Jacod and P. Protter. Risk neutral compatibility with option prices. Technical report, Université de Paris VI and Cornell University, April 2006.

[8] S. Kou. A jump-diffusion model for option pricing. Management Science, 48(8):1086 - 1101, 2002.

[9] D. Madan and E. Seneta. The Variance Gamma (V.G.) model for share market returns. Journal of Business, 63(4):511 - 24, 1990.

[10] Y. Ma R. Carmona and S. Nadtochiy. Calibration and simulation of arbitrage free implied volatility dynamic models. Technical report, Princeton University, 2010.

[11] P. Schönbucher. A market model for stochastic implied volatility. Phil. Trans. of the Royal Society, Series A, 357:2071 - 2092, 1999.

[12] M. Schweizer and J. Wissel. Term structures of implied volatilities: Absence of arbitrage and existence results. Mathematical Finance, (to appear), 2006.

[13] M. Schweizer and J. Wissel. Arbitrage-free market models for option prices: The multi-strike case. Technical report, 2007. 